\title{
Efficiency Improvement of Regenerative Energy for an EV
}

\author{
Takuya Yabe ${ }^{1}$, Kan Akatsu ${ }^{1}$, Nobunori Okui ${ }^{2}$, Tetsuya Niikuni ${ }^{2}$ and Terunao Kawai ${ }^{2}$ \\ ${ }^{1}$ Shibaura Institute of Technology \\ ${ }^{2}$ National Traffic Safety and Environment Laboratory
}

\begin{abstract}
Electric Vehicles (EVs) and various Hybrid Electric Vehicles (HEVs) have been attracting a lot of attention for environmental issues and energy crisis. One of advantages of using foregoing vehicles is charging energy by the regenerative brake. The running distance by one electric charge is increased a lot by the regenerative brake. However, the absorbed capacity of the regenerative energy is limited because of the motor capacity and the current limit of the battery. As a result not only the regenerative electric brake but also the mechanical brake must be used. This becomes serious issue in the heavy weight vehicle such as the bus and the truck, the effectiveness of EV/HEV is not obtained. To increase the regenerative energy, the large motor and the battery are requested, however, it is very difficult because of the cost and the limit of the inverter capacity.

In this paper, it is verified that the regenerative energy is increased by improving a braking method, averaging the deceleration, without changing the power train system. The proposed method is experimentally evaluated by $\mathrm{i}-\mathrm{MiEV}$ on the dynamo system, and increases the regenerative energy to $18 \%$.
\end{abstract}

Keywords: EV (electric vehicle), HEV (hybrid electric vehicle), regenerative brake,

\section{Introduction}

Recent warning of the exhausted fuel source and the global warming are serious problems in the world. EVs and HEVs are expected to reduce $\mathrm{CO}_{2}$ and almost every car manufactures are developing them. Although EVs are attractive to reduce $\mathrm{CO}_{2}$, it should increase the running distance by one electric charge to expand its market. To solve this problem, recent commercial EVs can absorb the regenerative energy as much as they can.

The brake system of EVs and HEVs is shown in Figure 1. A regenerative brake is achieved by using the traction motor which generates the negative torque in vehicle deceleration mode, it can convert the kinetic energy into the electric energy and it is charged to a battery, however, a general mechanical brake ingenerates the stopping force by overbearing brake disk to brake pad, the braking energy is converted into the heat and it is not reused. Then, the regenerative brake has little detriment and the running distance by one electric charge is increased by the regenerative brake. However, the possible absorbed energy of the regenerative energy is limited because of the motor capacity and the current limit of the battery. As a result not only the regenerative electric brake but also the mechanical brake must be used. The trend becomes serious for the heavy weight vehicles, the bus and the truck and so on.

However, in the research area of the brake system for EVs and HEVs, studies have been mostly focused on controlling the braking force to achieve the optimal braking performance [1]-[3]. Recent some studies have dealt with the optimal design of the brake system for EVs and HEVs [4]-[6]. However, few publications have experimentally verified an improvement of the regenerative energy by the brake system.

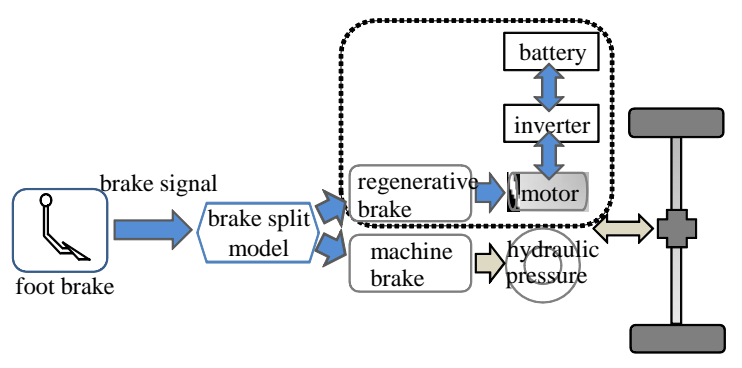

Figure 1: Brake system

In this paper, the reason why the regenerative energy is limited is firstly shown, and an improve 
method is proposed. The proposed method increases the regenerative energy by improving a breaking method. It is experimentally evaluated by actual i-MiEV on the dynamo system. The proposed method is possible to use to improve the regenerative energy in the heavy weight vehicles.

\section{Simulation model}

First, a vehicle simulation model is created by Matlab/Simulink which is shown in Figure 2 to make clear the effect of the motor capacity and the battery current to the regenerating energy. In this paper, a simple model that the vehicle differential equation is connected with the motor differential equation by the slip is made to understand the basis of phenomenon. Where, it assumes the ideal condition, that is, a bobbing motion, a pitching movement and a rolling movement are not taken into account and it is also assumed that the vehicle has one motor connected with the gear which ratio is selectable.

The motor differential equation is defined as follow.

$J \frac{d \omega}{d t}=T_{e}-F r-B \omega$

Where $J$ is the inertia force, $\omega$ is motor angular speed, $F$ is driving force, $r$ is wheel radius and $B$ is friction factor.

The vehicle motion differential equation is defined as follow.

$M \frac{d v}{d t}=F-F_{r}$

Where $M$ is the vehicle mass, $v$ is vehicle speed, $F_{r}$ is friction force, for example air friction and the rolling resistance. The friction factor between the road and the wheel associates equation (1) with (2). It is defined as equation (3) by the slip ratio. The slip ratio is defined as equation (4).

$\mu=C_{u}\left(\exp \left(-C_{a} S_{l}\right)-\exp \left(-C_{b} S_{l}\right)\right)$

$S_{l}=\frac{\omega r-v}{\omega r}$

Where, $C_{u}, C_{a}$ and $C_{b}$ are coefficients to determine the friction factor. The driving force is defined as follow.

$$
F=\mu M g
$$

The simple vehicle simulation model is made from the equation (1)-(5). The demand torque and power are calculated by inputting a speed reference to the speed controller and the torque reference is outputted to the vehicle model in which the output torque is equal to the reference.

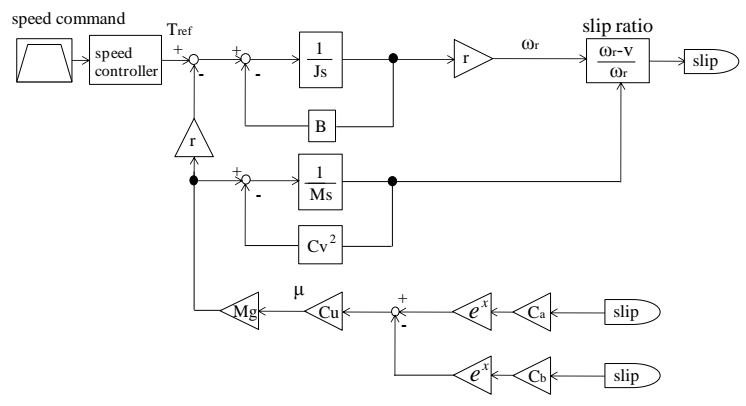

Figure 2: Vehicle model

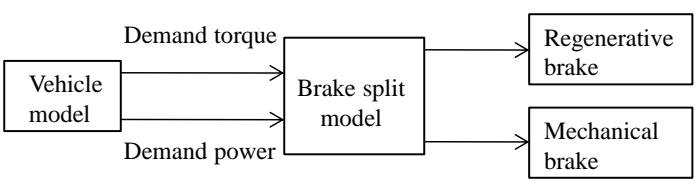

Figure 3: Brake model

The brake model is shown in Figure 3. The brake force is divided into the mechanical brake and the regenerative brake by the brake split model. The mechanical brake force is defined as a value subtracts the regenerative brake force from the demand brake command.

\section{Limitation of the regenerative brake}

The regenerative brake torque is calculated by the vehicle and the brake models. To make sure of the limitation of the regenerative brake, a heavy HEV model is calculated, and its parameter is shown in Table I.

Table I: Vehicle parameter

\begin{tabular}{|l|r|}
\hline Vehicle mass $(\mathrm{t})$ & 6 \\
\hline Motor max torque $(\mathrm{Nm})$ & 350 \\
\hline Motor max power $(\mathrm{kW})$ & 35 \\
\hline final gear ratio & 5.428 \\
\hline
\end{tabular}

Figure 4 shows a calculated result of the torque distribution between the mechanical brake and the electric regenerative brake when the heavy HEV is decelerated from $100 \mathrm{~km} / \mathrm{h}$ by $0.1 \mathrm{G}$ constant deceleration. The electric regenerative brake is limited because of the motor capacity, $35 \mathrm{~kW}$, at the high speed range, adding that even in the low speed range it is limited because of the input current limit of the battery. As a result, less than half of the braking energy is absorbed by the regenerative brake. 


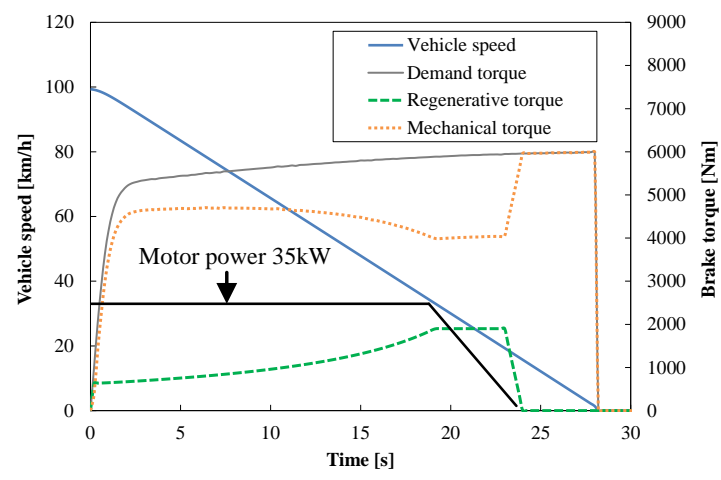

Figure 4: Distribution of the mechanical and the regenerative brake

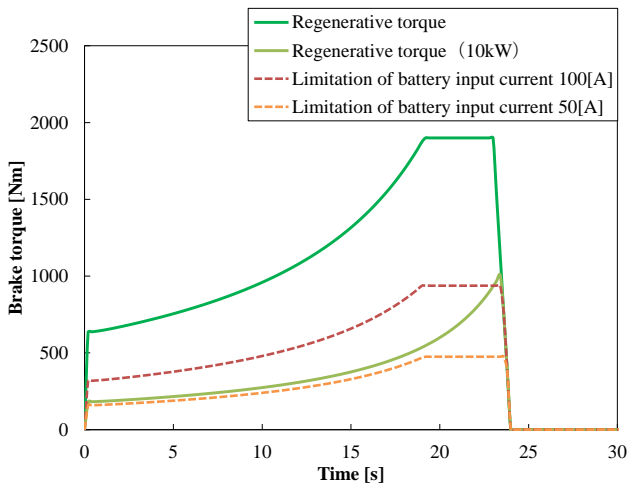

Figure 5: Regenerative brake torque of each capacity of the motor and the maximum input current into battery

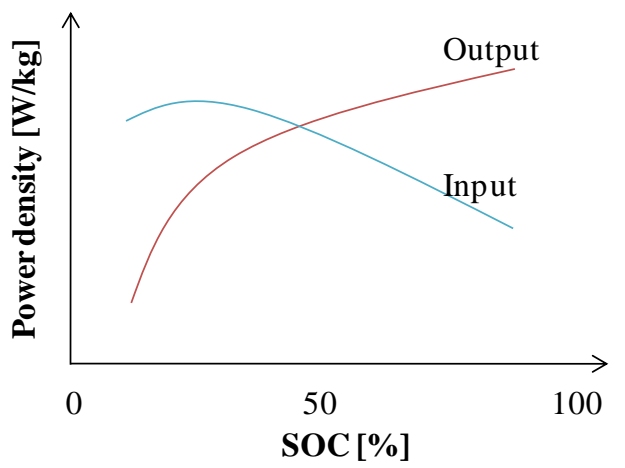

Figure 6: Input / Output property of battery

Figure 5 shows the study of the motor capacity and the battery input current limit. From this figure, it is observed that not only the motor capacity but also the battery input current effect to the electrical brake limit.

The maximum battery current is defined as follow.

$I_{\max . b a t}=\frac{P_{b} M_{b}}{V_{b a t}}$

Where, $I_{\max , b a t}$ is the maximum battery current, $P_{b}$ is battery current density, $M_{b}$ is battery mass per one cell and $V_{b a t}$ is battery voltage per one sell. Figure 6 shows Input / Output property of battery. At the high SOC condition the current input density is decreased. As a result the regenerative brake is limited by the maximum battery current.

Especially for the heavy EVs, HEVs like a bus and a truck, it is very hard to increase the battery capacity because of a trade off between the weight and the power. Also the motor capacity is hard to increase because of the cost and the limit of the inverter capacity.

\section{A proposed method to increase the amount of the regenerative energy}

The regenerative brake of EVs, HEVs is limited because of the motor capacity and the current limit of the battery as described above section. As a result not only the regenerative electric brake but also the mechanical brake must be used. To increase the regenerative energy, the motor capacity and the battery capacity are requested to be large, however it is very difficult because of the cost and the limit of the inverter capacity.

Therefore, in this paper, the regenerative energy is increased by improving a breaking method without changing the power train system.

\subsection{Averaging the deceleration method}

As an example JC08 mode which is used for the fuel consumption measurement in Japan is used as shown in Figure 7. The proposed method is averaging the deceleration when the car is decelerated. Figure 8 shows a comparison of the deceleration between the JC08 model and the proposed model. The deceleration of the proposed model is smaller than the JC08 model by averaging the deceleration.

Figure 9, 10 and 11 show the comparison results of the vehicle speed, the deceleration and the demand brake power, respectively. Figure 10 shows the deceleration of the proposed model is half of the maximum value of the original model by averaging the deceleration. As a result not only the deceleration but also the max demand power is reduced two-thirds value. As a result the regenerative energy of the proposed model is larger than the JC08 model even when the regenerative brake is limited by the input current limit of the battery. Averaging the deceleration method can be achieved by using a driving assistance tool, for example, the deceleration signal is shown on a display with the navigation which gives the advice to the driver on the optimal 
vehicle operation [7], or controlling a brake pedal by applying a brake system like an active accelerator pedal, which indicates the ratio of acceleration of the driver [8].

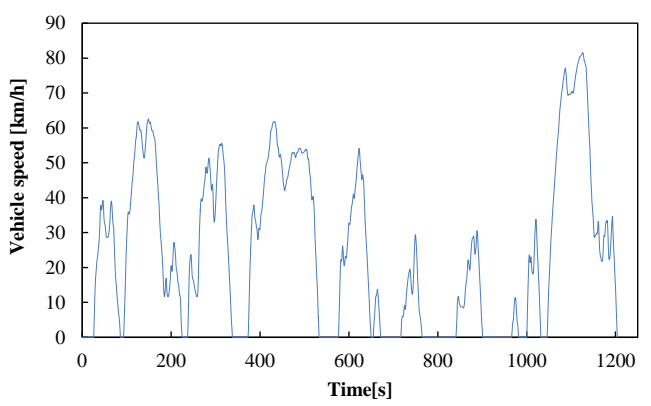

Figure 7: JC08mode

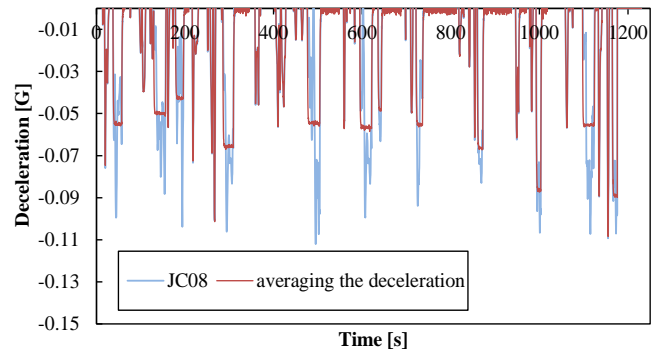

Figure 8: Deceleration

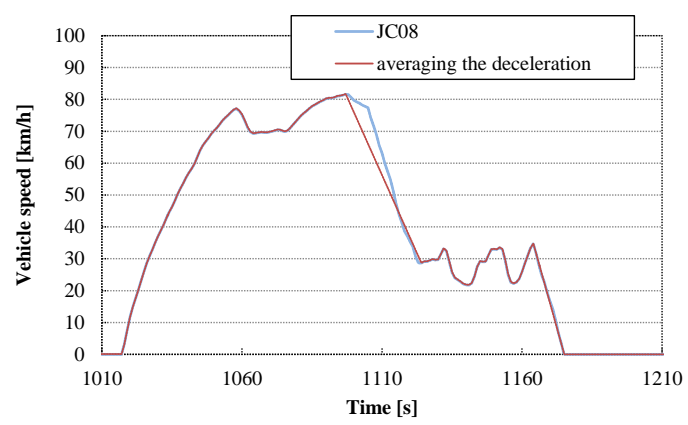

Figure 9: The comparison of the vehicle speed

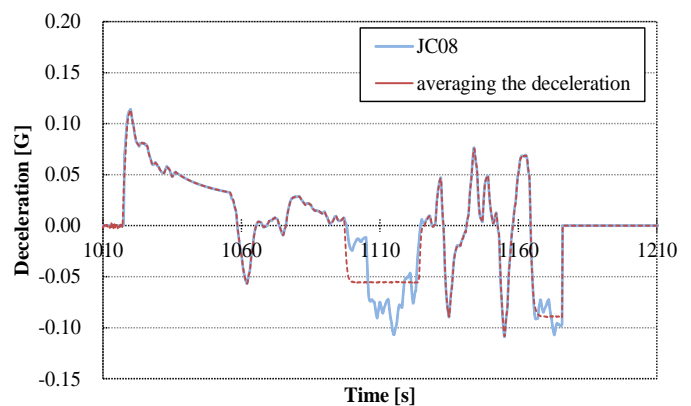

Figure 10: The comparison of the deceleration

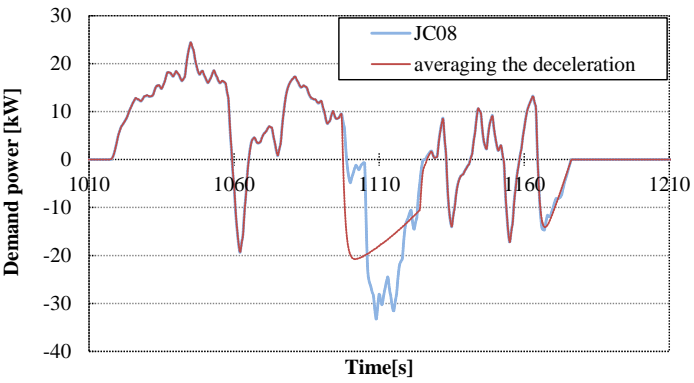

Figure 11: The comparison of the demand brake power

\subsection{Experimental equipments}

In the experiment to evaluate the proposed method, "i-MiEV" is used to measure the regenerative electric power at the real condition. Table II shows the vehicle parameter, and both Figure 12 and 13 show the experimental equipments. The proposed method is evaluated by running $\mathrm{i}-\mathrm{MiEV}$ on the dynamo system. The experiment by the dynamo system is taken account of the air resistance and the rolling friction resistance by pre-measured.

Table II: Vehicle parameter

\begin{tabular}{|lc|c|}
\hline Vehicle mass $\quad(\mathrm{kg})$ & 1110 \\
\hline Width $\quad(\mathrm{mm})$ & 1475 \\
\hline Height $\quad(\mathrm{mm})$ & 1610 \\
\hline Battery voltage $\quad(\mathrm{V})$ & 330 \\
\hline Motor max power $\quad(\mathrm{kW})$ & 47 \\
\hline Motor max torque $\quad(\mathrm{Nm})$ & 180 \\
\hline Final gear ratio & 6.066 \\
\hline Wheel radius $\quad(\mathrm{m})$ & 0.2865 \\
\hline
\end{tabular}

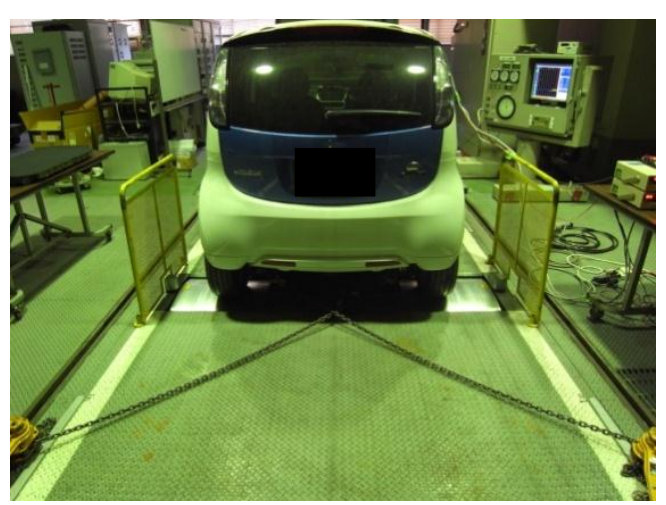

Figure 12: "i-MiEV" on the dynamo system 


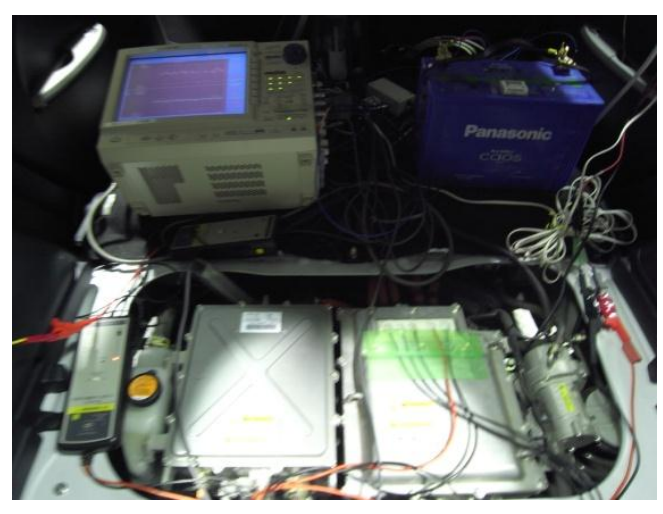

Figure 13: Battery of "i-MiEV"

\subsection{Experimental results}

\subsubsection{Experimental result}

Figure 15 shows the motor current, the accelerating current is larger than the decelerating one because the limitation of the regenerative torque. It is confirmed that the regenerative torque is limited to about $20 \mathrm{Nm}$ in Figure 16. The detail is shown in the next section.

Figure 17 shows the battery voltage of both methods. The voltage in the proposed model is smaller than it in the JC08 model because of the influence of the SOC condition.

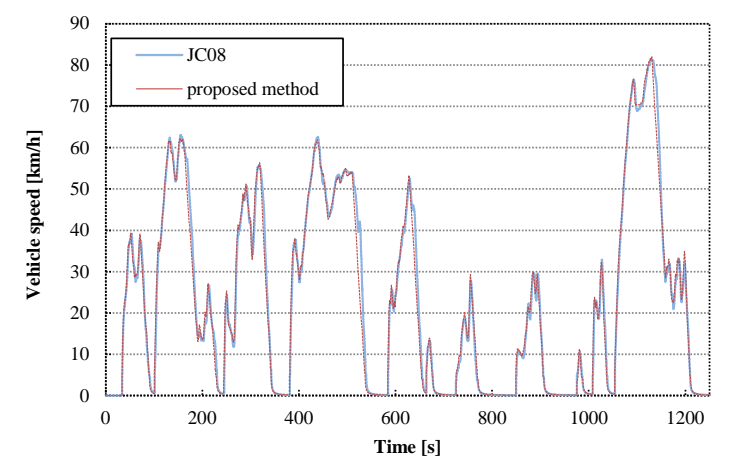

Figure 14: Vehicle speed

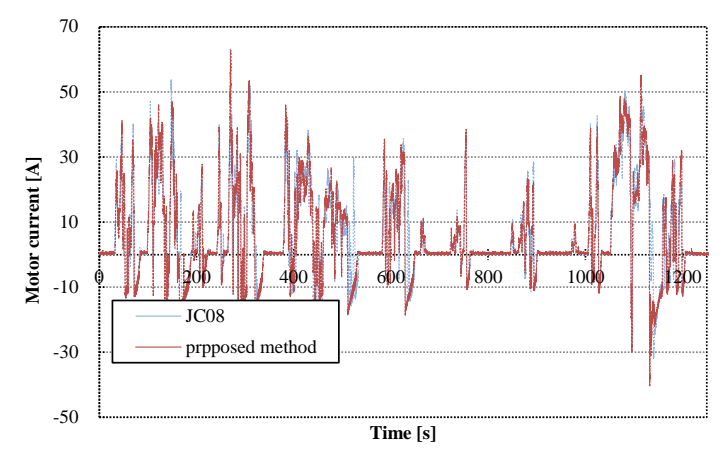

Figure 15: Motor current

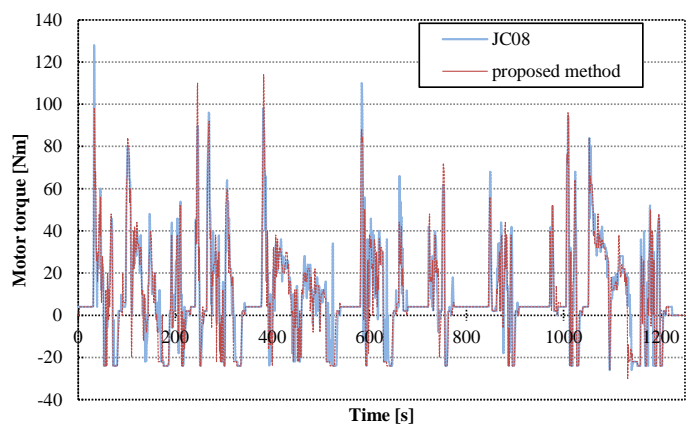

Figure 16: Motor torque

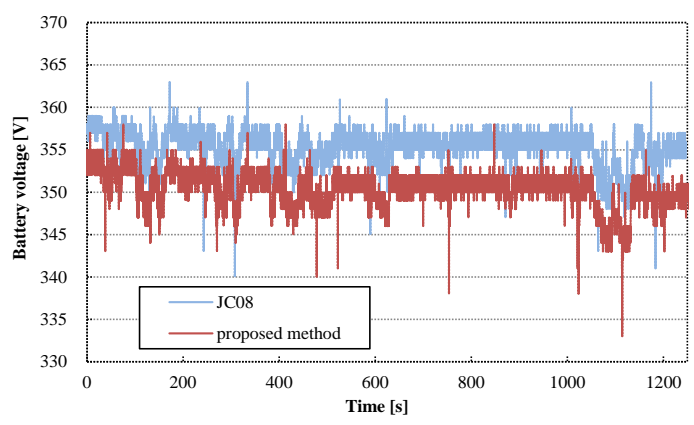

Figure 17: Battery voltage

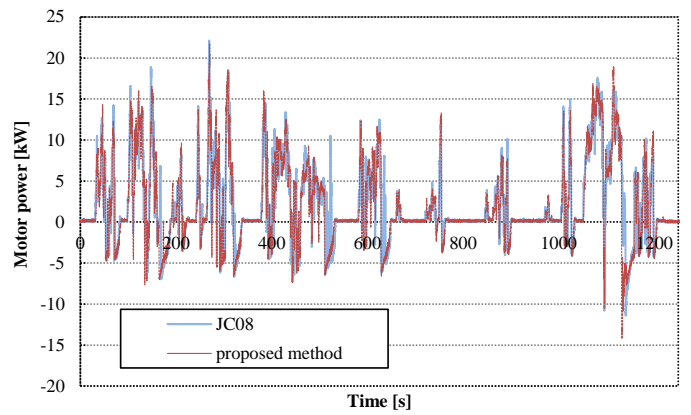

Figure 18: Motor power

\subsubsection{Limitation of the regenerative brake}

The experimented result of the motor torque with decelerating is shown in Figure 19. The regenerative torque is limited to $24 \mathrm{Nm}$. This is because "i-MiEV" does not control the regeneration brake associated with the mechanical brake, that results difficulty of the precise braking control, it means the regeneration brake is worked every when the driver release the acceleration pedal. Then to keep the safety also to avoid much de-acceleration the regenerative torque is limited.

Figure 20 shows the comparison between the demand power and the regenerative power. Less than half of the braking energy is absorbed by the regenerative brake. As a result the mechanical brake is much used. 


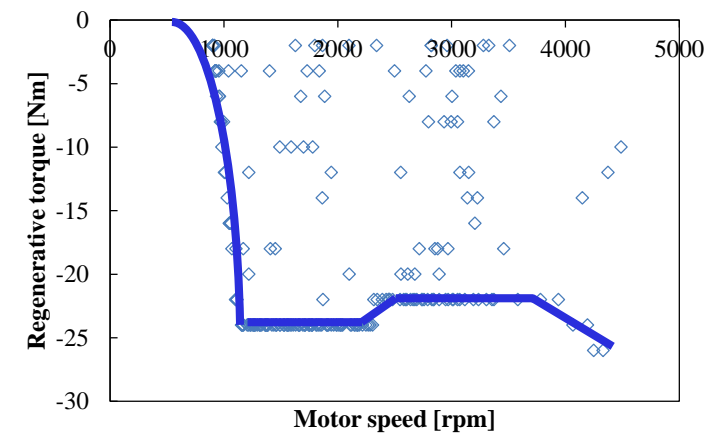

Figure 19: Regenerative braking torque of motor

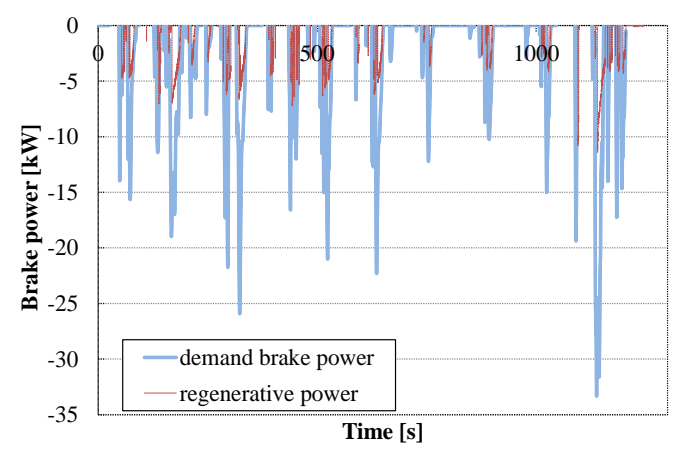

Figure 20: Comparison of demand power and regenerative power

\subsubsection{Regenerative limitation in low speed range}

Figure 21 shows the regenerative limit in the low speed range. From this figure it is confirmed that the regeneration is completely terminated, the current becomes zero under the speed is bellow $16 \mathrm{~km} / \mathrm{h}$. As described above, that is because the regeneration control in the low speed range is sensitive, adding that the motor voltage becomes small in the low speed region.

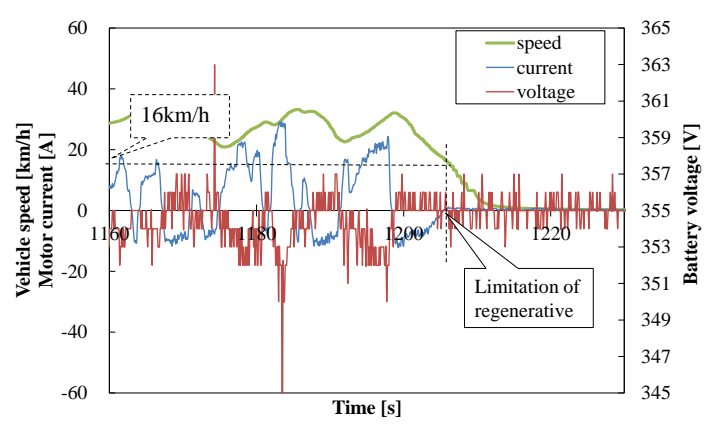

Figure 21: Limitation of regenerative brake in low speed range

\subsubsection{Comparison of the regenerative energy}

The motor power is compared in each running modes. Figure 22 shows an expansion of the motor input/output power comparison between the proposed model and the original model. The regenerative power in the proposal model is much absorbed than in JC08 mode especially when the deceleration is changed with smooth deacceleration.

Figure 23 shows a comparison of the regenerative energy. Three times experimental results are averaged and compared with the original model. The regenerative energy is increased $18 \%$ by the proposed averaging deceleration method.

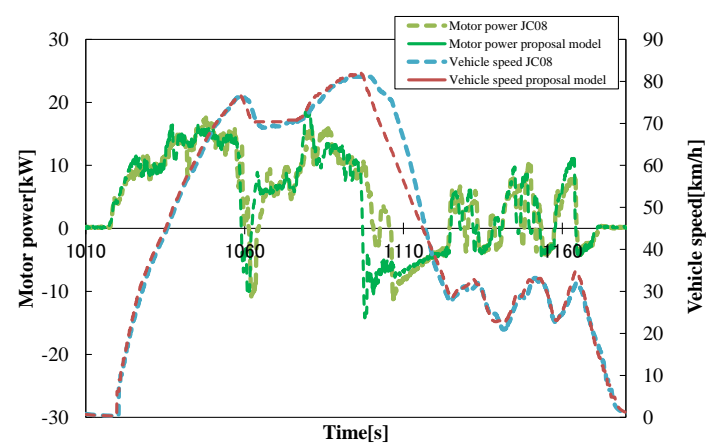

Figure 22: Comparison of motor power

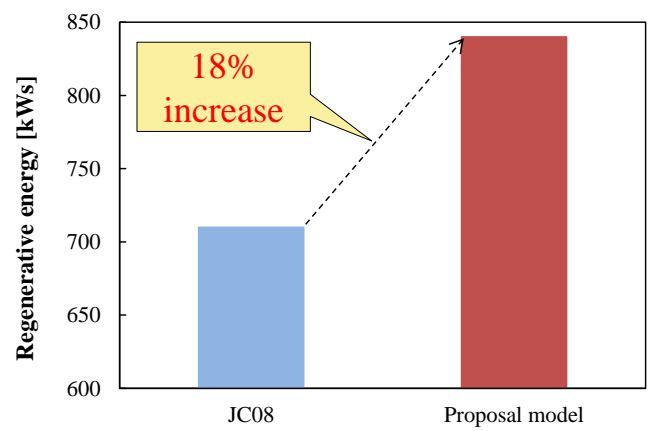

Figure 23: Comparison of the amounts of the absorbed regenerative electric power

\section{Conclusion}

In this paper, the reason why the regenerative energy is limited is firstly shown, and an improve method to absorb the regenerative energy is proposed. The proposed method is experimentally evaluated by i-MiEV on the dynamo system, and increases the regenerative energy $18 \%$. The proposed method is realized by the driving operation, the regenerative energy is much increased, for example it is effective to adapt to the 
public bus because a bus lane allows the optimal deceleration to absorb the regenerative energy.

\section{References}

[1] Chunting Mi, Hui Lin and Yi Zhang, "Iterative Learning Control of Antilock Barking of Electric and Hybrid Vehicles", IEEE Transaction on Vehicular Technology, Vol. 54, No. 2, Sep. 2005

[2] Nobuyoshi Mutoh, Hiromichi Yahagi, "Electrical Braking Control Methods for Electric vehicles with Independently Driven Front and Rear Wheel Structure", Industrial Electronics, IEEE transaction on, Vol. 54, Issue 2. April 2007

[3] Li Yu-shan, Zeng Qing-liang, Wang Chenglong, Wang Liang, "Research on Control strategy for Regenerative Braking of a Plug in Hybrid Electric City Public Bus", IEEE Intelligent Computation Technology and Automation, Oct. 2009

[4] Yimin Gao, Liang Chu, Mehrdad Ehsani, "Design and Control Principles of Hybrid Braking System for EV, HEV and FCV", IEEE Vehicle Power and Propulsion Conference, Sept. 2007,

[5] Liang Chu, Wanfeng Sun, Liang Yao, Yongsheng Zhang, Yang Ou, Wenruo Wei, Minghui Liu, Jun Li, "Integrative control strategy of regenerative and hydraulic braking for hybrid electric car ", IEEE Vehicle Power and Propulsion Conference, Oct. 2009,

[6] S. R. Clikanek, K. E. Bailey, "Regenerative Braking System for A Hybrid Electric Vehicle", Proceedings of the American Control Conference, Anchorage, AK May, 2002,

[7] M. van der Voort, M. Dougherty, "Reducing fuel consumption by using a new fuel-efficiency support tool", IEEE Africon, Sep.1999,

[8] A. Varhelyi, M. Hjalmdahl, C. Hyden, and M. Draskoczy, "Effects of an active accelerator pedal on driver behaviour and traffic safety after long-term use in urban areas", Accident Analysis and Prevention, vol. 36 no. 5, pp. 729-737, 2004, 